\title{
Preparation of Optically Pure L-2-Hydroxyaldehydes with Yeast Transketolase ${ }^{1}$
}

\author{
Franz Effenberger*, Volker Null and Thomas Ziegler \\ Institut für Organische Chemie, Universitat Stuttgart, Pfaffenwaldring 55, W-7000 Stuttgart 80, Germany
}

\begin{abstract}
L-2-Hydroxyaldehydes L-3 with a great variety of substituents in 3-position are obtained in good chemical and excellent optical yields by kinetic resolution in the transketolase-catalyzed reaction of racemic 2-hydroxyaldehydes with lithium hydroxypyruvale 4 where only the enantiomer $(R)-3$ reacts to 5 -deoxy-D-xyluloses 5 .
\end{abstract}

Exclusively the $(R)$-enantiomer of racemic 3-azido-2-hydroxypropanal reacts with lithium hydroxypyruvate 4 and yeast transketolase (EC 2.2.1.1) as catalyst to 5 -azido-5-deoxy-D-xylulose; ${ }^{2}$ from the reaction mixture the $(S)$-enantiomer can be isolated in good chemical yield and high optical purity. ${ }^{2}$ A kinetic resolution of racemic 2-hydroxyaldehydes, which are interesting compounds for various syntheses, ${ }^{3}$ with transketolase as catalyst therefore can be reached. Transketolase-catalyzed kinetic separations of racemic 2hydroxyaldehydes were mentioned in the literature, ${ }^{4}$ but were not yet used for the preparation of optically active $\alpha$-hydroxyaldehydes.

In generally, optically active 2-hydroxyaldehydes can be synthesized by hydrogenation of the corresponding 2-hydroxycarboxylic acid derivatives. ${ }^{5}$ Several optically active 2-hydroxyaldehydes were obtained as their diethyl acetals via lipase-catalyzed kinetic resolution of racemic 2 -acetoxyaldehyde diethyl acetals. ${ }^{6}$ Optically pure glyceraldehydes, important starting compounds for many syntheses, ${ }^{3}$ were obtained from natural sources. For example $(R)-2,3-O$-isopropylidene glyceraldehyde is accessible from D-mannitol and the corresponding $(S)$-enantiomer starting from L-ascorbic acid. ${ }^{7}(R)$ - and (S)-2-O-benzyl glyceraldehyde resp. which are less sensible to racemization can be obtained from the esters of D- or L-tartaric acid. ${ }^{8}$

From the racemic 2 -hydroxyaldehydes $3 \mathrm{a}-\mathrm{g}$, used for the transketolase-catalyzed reactions, the aldehydes $3 \mathbf{a}, 3 \mathrm{c}$, and $\mathbf{3 g}$ have not yet been described in the literature; they were obtained by regioselective ring opening of the easily accessible 2-(diethoxymethyl)oxirane ${ }^{9} 1$ with the respective nucleophiles $\mathrm{R}^{-} \mathrm{M}^{+}$(Table 1 ).

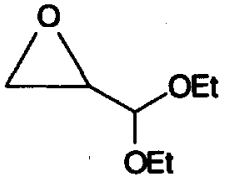

1<smiles>[R]CC(O)C(OCC)OCC</smiles>

2<smiles>[R]CC(O)C=O</smiles>

3

The regioselectivity of the oxirane ring opening is influenced by electronic and steric effects. ${ }^{10}$ With strong nucleophiles ring opening of 1 occurs exclusively in 3-position to give the acetals 2 which can be isolated and characterized. The hydroxyaldehydes 3 itself, however, received by acid catalyzed hydrolysis 
from compounds $\mathbf{2}$ are obtained in aqueous solution as hydrates or as colorless dimers, for example $\mathbf{3 a}$ and $3 \mathrm{~g}$, whose structure has been established by mass spectrometry. ${ }^{11}$

Table 1. Yields of Diacetals 2 and Hydroxyaldehydes 3 by Nucleophilic Ring Opening of the Oxirane 1 and Subsequent Hydrolysis

\begin{tabular}{|llllll|}
\hline & & & 2 & & 3 \\
$\mathrm{R}^{-}$ & $\mathbf{M}^{+}$ & & yield [\%] & & yield [\%] \\
\hline $\mathrm{PhCH}_{2} \mathrm{O}$ & $\mathrm{Na}$ & $\mathbf{a}$ & 45 & $\mathrm{a}$ & $85^{\mathrm{a}}$ \\
$\mathrm{HS}$ & $\mathrm{K}$ & $\mathbf{c}$ & 95 & $\mathbf{c}$ & 92 \\
$\mathrm{CN}$ & $\mathrm{K}$ & $\mathrm{g}$ & 60 & $\mathrm{~g}$ & $96^{\mathrm{a}}$ \\
\hline
\end{tabular}

a Isolated as dimers.

Racemic 2-hydroxyaldehydes 3a-g react with lithium hydroxypyruvate 4 and yeast transketolase (EC 2.2.1.1) as catalyst to give the L-2-hydroxyaldehydes L-3a,b,d-g and the corresponding 5-substituted 5deoxy-D-xyluloses 5a-g in good chemical yields and in excellent optical purity (Table 2).<smiles>[13CH3]OC(=O)C(=O)CO</smiles>

4

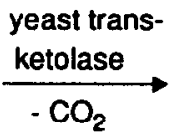

$L-3$<smiles>[R]C[C@@H](O)C=[O+][C@H](O)[C@@H](O)C(=O)CO</smiles>

5

Table 2. Yeast Transketolase (TK)-Catalyzed Reaction of Racemic 2-Hydroxyaldehydes 3a-g with Equimolar Amounts of Lithium Hydroxypyruvate $4^{12}$

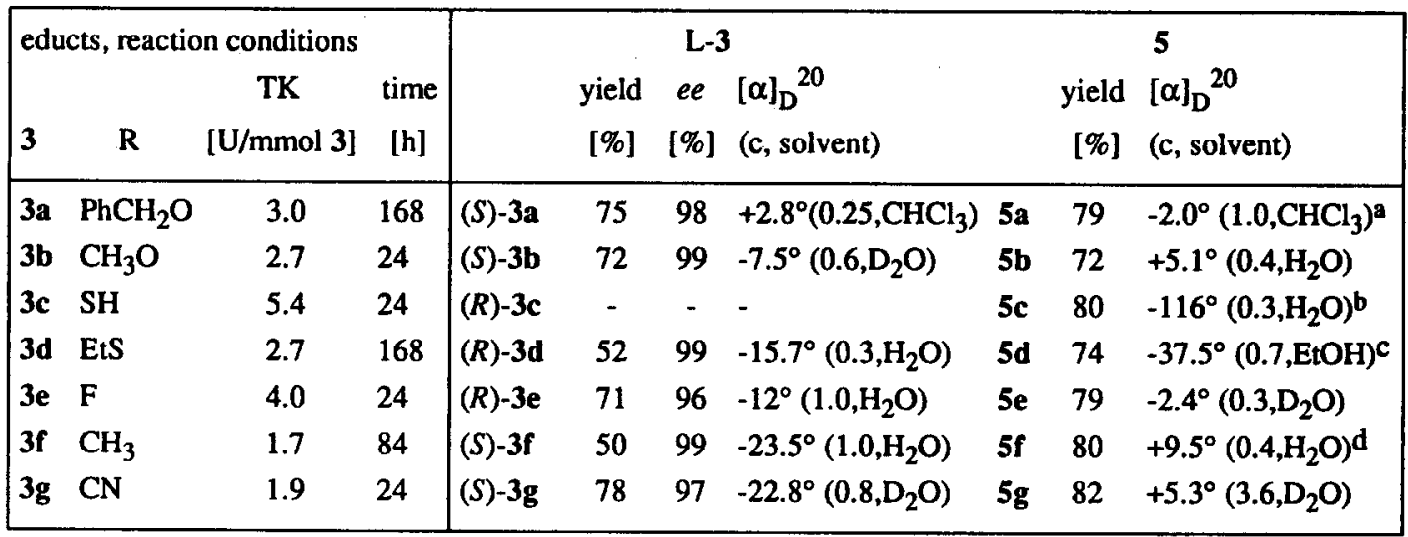

a) $\left.\left.[\alpha]_{D}^{20}=-2.2^{\circ}\left(c=0.98, \mathrm{CHCl}_{3}\right) .{ }^{13} \mathrm{~b}\right)[\alpha]_{D}{ }^{20}=-103.8^{\circ}\left(\mathrm{c}=3.23, \mathrm{D}_{2} \mathrm{O}\right){ }^{14} \mathrm{c}\right)[\alpha]_{\mathrm{D}}{ }^{20}=-41^{\circ}(\mathrm{c}=0.9, \mathrm{EtOH}) .1^{15}$

d) $[\alpha]_{D}{ }^{20}=+6^{\circ}\left(\mathrm{c}=2.5, \mathrm{H}_{2} \mathrm{O}\right){ }^{16}$ 
The highest conversion in the yeast transketolase-catalyzed reaction is achieved with lithium hydroxypyruvate 4 and racemic 2-hydroxyaldehydes 3 in equimolar ratio. Despite a partly deactivation of the enzyme, a subsequent addition of transketolase is not necessary. The course of the resolution of the racemic aldehydes 3 was followed either by derivatization $(3 \mathbf{3 a}, \mathrm{b}, \mathrm{d}-\mathrm{g})$ or by measuring the optical rotation (3c). After incubation at $30^{\circ} \mathrm{C}$ at the $\mathrm{pH}$ optimum 7.6 of the enzyme ${ }^{17}$ the reactions were started by addition of the enzyme. The reactions were stopped by addition of Dowex $50 \mathrm{WX8 \textrm {H } ^ { + }}$ and Dowex $1 \mathrm{X8} \mathrm{HCO}_{3}^{-}$when no change of concentration of 4 nor a change of $\alpha_{D}$ for L-3 is observed. The pure 2-hydroxyaldehydes L-3b,d-g and the 2-ketoses 5 were isolated by chromatography on Dowex $50 \mathrm{WX8} \mathrm{Ca}^{2+}$. In case of the reaction of $3 \mathrm{c}$ only the ketose $5 \mathrm{c}$ but not L-3c could be isolated. (S)-3a and $5 \mathrm{a}$ were obtained by extraction and perforation with diethyl ether or ethyl acetate followed by column chromatography on silica gel. The L-hydroxyaldehydes L-3 were converted into the corresponding diethyl acetals and the enantiomeric excess was determined by gas chromatography on a $\beta$-cyclodextrine phase. The enantiomerically pure hydroxyaldehydes $\mathrm{L}-3$ are oily compounds; they do not dimerize in aqueous solution in contrast to the racemic aldehydes 3 .

Acknowledgement: This work was generously supported by the Deutsche Forschungsgemeinschaft.

\section{References and Notes}

1. Enzyme-catalyzed Reactions, Part 12.- Part 11: Effenberger, F.; Stelzer, U. Angew. Chem. Int. Ed. Engl. 1991, 30, 873-874.

2. Ziegler, T.; Straub, A.; Effenberger, F. Angew. Chem. Int. Ed. Engl. 1988, 27, 716-717.

3. Mulzer, J. (R)- and (S)-2,3-Isopropylidene Glyceraldehyde - "Unbiased" Chiral Starting Materials. In Organic Synthesis Highlights; Altenbach, H.-J.; Braun, M.; Krohn, K.; Reissig, H. Eds.; VCH-Verlagsgesellschaft: Weinheim, 1991; pp. 243-250.

Bestmann, H.J.; Pecher, B.; Riemer, C. Synthesis 1991, 731-734.

4. Bolte, J.; Demuynck, C.; Samaki, H. Tetrahedron Lett. 1987, 28, 5525-5528.

Myles, D.C.; Andrulis III, P.J.; Whitesides, G.M. Tetrahedron Lett. 1991, 32, 4835-4838.

5. Massad, S.K.; Hawkins, L.D.; Baker, D.C. J. Org. Chem. 1983, 48, $5180-5182$.

Hoppe, D.; Tarara, G.; Wilckens, M. Synthesis 1989, 83-88.

Kobayashi, Y.; Takase, M.; Ito, Y.; Terashima, S, Bull. Chem. Soc. Jpn. 1989, 62, 3038-3040.

Effenberger, F.; Hopf, M.; Ziegler, T.; Hudelmayer, J. Chem. Ber. 1991, 124, 1651-1659.

6. Von der Osten, C.H.; Sinskey, A.J.; Barbas, C.F.; Pederson, R.L.; Wang, Y.-F.; Wong, C.-H. J. Am. Chem. Soc. 1989, 111, 3924-3927.

Pederson, R.L.; Liu, K.K.-C.; Rutan, J.F.; Chen, L.; Wong, C.-H. J. Org. Chem. 1990, 55, 4897-4901.

7. Fischer, H.O.L.; Baer, E. Helv. Chim. Acta 1936, 19, 519-532.

Dumont, R.; Pfander, H. Helv. Chim. Acta 1983, 66, 814-823.

Schmid, C.R.; Bryant, J.D.; Dowlatzedah, M.; Phillips, J.L.; Prather, D.E.; Schantz, R.D.; Sear, N.L.; Vianco, C.S. J. Org. Chem. 1991, 56, 4056-4058.

Takano, S.; Numata, H.; Ogasawara, K. Heterocycles 1982, 19, 327-328.

8. Jäger, V.; Wehner, V. Angew. Chem. Int. Ed. Engl. 1989, 28, 469-470.

9. Payne, G.B.; Deming, P.H.; Williams, P.H. J. Org. Chem. 1961, 26, 659-663.

10. Parker, R.E.; Isaacs, N.S. Chem. Rev. 1959, 59, 737-799.

Behrens, C.H.; Sharpless, K.B. Aldrichimica Acta 1983, 16, 67-79.

11. Racemic 3a (persilylated): MS (70 eV): Calc. 504.2363 Found $504.2360 ; \mathrm{m} / \mathrm{z}(\%): 504(0.06)\left[\mathrm{M}^{+}\right]$, $324(0.32)\left[\mathrm{M}^{+}-2\left(\mathrm{CH}_{3}\right)_{3} \mathrm{SiOH}\right], 281$ (2.6), 107 (11.2), $91(100)\left[\mathrm{C}_{7} \mathrm{H}_{7}{ }^{+}\right]$. Racemic $3 \mathrm{~g}$ (persilylated): MS (70 eV): Calc. 342.1431 Found 342.1430; m/2 (\%): $341(1.5), 191$ (18.4), $156(100)$.

12. Preparation of 2-hydroxyaldehydes $L-3$ and ketoses 5; general procedure: $A$ freshly prepared solution of the corresponding aldehyde $3 \mathrm{a}-\mathrm{g}, 4$ (each $25-50 \mathrm{mM}$ ), $\mathrm{MgCl}_{2}$ dihydrate and thiamine pyrophosphate in Tris- $\mathrm{HCl}$ buffer $(0.5 \mathrm{M}, \mathrm{pH} 7.6)$ were incubated at $30^{\circ} \mathrm{C}$. The reactions are started by addition of yeast transketolase (EC 2.2.1.1) (Table 2). After the given time (Table 2) the reaction mixture is desalted with Dowex $50 \mathrm{WX} 8 \mathrm{H}^{+}$and Dowex $1 \times 8 \mathrm{HCO}_{3}^{-}$. The solutions were concentrated and the products L-3 and 5 were separated by column chromatography on Dowex $50 \mathrm{WX} 8 \mathrm{Ca}^{2+}$ (column $3 \mathrm{~cm} \times$ $85 \mathrm{~cm}$ ) with water as eluent. $(S)-3 \mathrm{a}$ was reprocessed by extraction with diethyl ether and perforation of 
the aqueous phase with ethyl acetate within $48 \mathrm{~h}$. The combined organic phases were dried $\left(\mathrm{Na}_{2} \mathrm{SO}_{4}\right)$, concentrated and chromatographed on silica gel with methanol/dichloromethane/petroleum ether $(1+2+4)$. All compounds gave correct elemental analyses and were characterized by ${ }^{1}$ H-NMR spectroscopy.

1 $\mathrm{H}-\mathrm{NMR}$ data $(250 \mathrm{MHz}, 8)$

\begin{tabular}{|c|c|}
\hline$(S)-3 \mathbf{a}$ & $\begin{array}{l}1.76(\mathrm{~s}, 1 \mathrm{H}, \mathrm{OH}), 3.40-3.70 \text { and } 5.10-5.40(\mathrm{~m}, 4 \mathrm{H}, 1-, 2-, 3-\mathrm{H}), 4.54\left(\mathrm{~s}, 2 \mathrm{H}, \mathrm{CH}_{2} \mathrm{Ph}\right), 7.30-7.39(\mathrm{~m} \text {, } \\
5 \mathrm{H}, \mathrm{Ph})\end{array}$ \\
\hline$(S)-\mathbf{3 b}$ & $\begin{array}{l}3.40\left(\mathrm{~s}, 3 \mathrm{H}, \mathrm{CH}_{3}\right), 3.53\left(\mathrm{dd}, J_{2,3}=3.0 \mathrm{~Hz}, J_{3,3}=-10.5 \mathrm{~Hz}, 1 \mathrm{H}, 3-\mathrm{H}\right), 3.64\left(\mathrm{dd}, J_{2,3}=6.9 \mathrm{~Hz}, 1 \mathrm{H},\right. \\
\left.3-\mathrm{H}), 3.69 \text { (ddd, } J_{1,2}=5.3 \mathrm{~Hz}, 1 \mathrm{H}, 2-\mathrm{H}\right), 4.93(\mathrm{~d}, 1 \mathrm{H}, 1-\mathrm{H})\end{array}$ \\
\hline$(R)-3 \mathbf{d}$ & $\begin{array}{l}1.25\left(\mathrm{t}, J=7.3 \mathrm{~Hz}, 3 \mathrm{H}, \mathrm{CH}_{3}\right), 2.63\left(\mathrm{q}, 2 \mathrm{H}, \mathrm{CH}_{2} \mathrm{CH}_{3}\right), 2.65\left(\mathrm{dd}, J_{2,3}=8.5 \mathrm{~Hz}, J_{3,3}=-13.8 \mathrm{~Hz}, 1 \mathrm{H},\right. \\
3-\mathrm{H}), 2.88\left(\mathrm{dd}, J_{2,3}=3.6 \mathrm{~Hz}, 1 \mathrm{H}, 3-\mathrm{H}\right), 3.66\left(\mathrm{ddd}, J_{1,2}=5.1 \mathrm{~Hz}, 1 \mathrm{H}, 2-\mathrm{H}\right), 4.95(\mathrm{~d}, 1 \mathrm{H}, 1-\mathrm{H})\end{array}$ \\
\hline$(R)-3 \mathrm{e}$ & $\begin{array}{l}3.75\left(\text { dddd, } J_{\mathrm{F}, 2}=23.8 \mathrm{~Hz}, J_{2,3}=3.1 \mathrm{~Hz}, J_{2,3}=5.1 \mathrm{~Hz}, J_{1,2}=6.0 \mathrm{~Hz}, 1 \mathrm{H}, 2-\mathrm{H}\right), 4.58\left(\mathrm{dd}, J_{3,3}=-10.2\right. \\
\left.\mathrm{Hz}, J_{\mathrm{F}, 3}=47.2 \mathrm{~Hz}, 1 \mathrm{H}, 3-\mathrm{H}\right), 4.63(\mathrm{dd}, 1 \mathrm{H}, 3-\mathrm{H}), 5.00(\mathrm{~d}, 1 \mathrm{H}, 1-\mathrm{H})\end{array}$ \\
\hline$(S)-3 f$ & $0.96\left(\mathrm{t}, 3 \mathrm{H}, \mathrm{CH}_{3}\right), 1.40-1.80\left(\mathrm{~m}, 2 \mathrm{H}, \mathrm{CH}_{2}\right), 3.40-3.90(\mathrm{~m}, 1 \mathrm{H}, 2-\mathrm{H}), 4.30-5.30(\mathrm{~m}, 1 \mathrm{H}, 1-\mathrm{H})$ \\
\hline$(S)-3 g$ & $\begin{array}{l}2.74\left(\mathrm{dd}, J_{2,2}=-17.2 \mathrm{~Hz}, J_{2,3}=7.2 \mathrm{~Hz}, 1 \mathrm{H}, 2-\mathrm{H}\right), 2.84\left(\mathrm{dd}, J_{2,3}=4.5 \mathrm{~Hz}, 1 \mathrm{H}, 2-\mathrm{H}\right), 3.86 \text { (dddd, } \\
\left.J_{3,4}=5.3 \mathrm{~Hz}, 1 \mathrm{H}, 3-\mathrm{H}\right), 4.97(\mathrm{~d}, 1 \mathrm{H}, 4-\mathrm{H})\end{array}$ \\
\hline $5 a$ & $\begin{array}{l}1.70,2.70,3.00(3 \mathrm{~s}, 3 \mathrm{H}, \mathrm{OH}), 3.60-3.70(\mathrm{~m}, 2 \mathrm{H}, 5-\mathrm{H}), 4.12(\mathrm{~m}, 1 \mathrm{H}, 4-\mathrm{H}), 4.33 \text { and } 4.41(\mathrm{AB} \\
\text { system, } 2 \mathrm{H}, 1-\mathrm{H}), 4.40(\mathrm{~d}, 1 \mathrm{H}, 3-\mathrm{H}), 4.55\left(\mathrm{~s}, 2 \mathrm{H}, \mathrm{CH}_{2} \mathrm{Ph}\right), 7.30-7.40(\mathrm{~m}, 5 \mathrm{H}, \mathrm{Ph})\end{array}$ \\
\hline $5 b$ & $\begin{array}{l}3.38\left(\mathrm{~s}, 3 \mathrm{H}, \mathrm{CH}_{3}\right), 3.53\left(\mathrm{dd}, J_{4,5}=7.2 \mathrm{~Hz}, J_{5,5}=-10.4 \mathrm{~Hz}, 1 \mathrm{H}, 5-\mathrm{H}\right), 3.60\left(\mathrm{dd}, J_{4,5}=5.2 \mathrm{~Hz}, 1 \mathrm{H} \text {, }\right. \\
5-\mathrm{H}), 4.18\left(\mathrm{ddd}, J_{3,4}=2.5 \mathrm{~Hz}, 1 \mathrm{H}, 4-\mathrm{H}\right), 4.39(\mathrm{~d}, 1 \mathrm{H}, 3-\mathrm{H}), 4.50,4.60 \text { (AB syst., } J_{1,1}=-19.4 \mathrm{~Hz} \text {, } \\
2 \mathrm{H}, 1-\mathrm{H})\end{array}$ \\
\hline $5 c$ & $\begin{array}{l}2.63\left(\mathrm{dd}, J_{4,5}=9.2 \mathrm{~Hz}, J_{5,5}=-10.3 \mathrm{~Hz}, 1 \mathrm{H}, 5-\mathrm{H}\right), 3.08\left(\mathrm{dd}, J_{4,5}=7.3 \mathrm{~Hz}, 1 \mathrm{H}, 5-\mathrm{H}\right), 3.66(\mathrm{~s}, 2 \mathrm{H}, \\
1-\mathrm{H}), 3.80\left(\mathrm{~d}, J_{3.4}=9.4 \mathrm{~Hz}, 1 \mathrm{H}, 3-\mathrm{H}\right), 4.34(\mathrm{ddd}, 1 \mathrm{H}, 4-\mathrm{H})\end{array}$ \\
\hline 5d & $\begin{array}{l}1.22\left(\mathrm{t}, J=7.4 \mathrm{~Hz}, 3 \mathrm{H}, \mathrm{CH}_{3}\right), 2.60\left(\mathrm{q}, 2 \mathrm{H}, \mathrm{CH}_{2}\right), 2.70\left(\mathrm{dd}, J_{4.5}=7.5 \mathrm{~Hz}, J_{5,5}=-13.8 \mathrm{~Hz}, 1 \mathrm{H}, 5-\mathrm{H}\right) \text {, } \\
2.73\left(\mathrm{dd}, J_{4,5}=6.6 \mathrm{~Hz}, 1 \mathrm{H}, 5-\mathrm{H}\right), 4.13\left(\mathrm{ddd}, J_{3,4}=2.2 \mathrm{~Hz}, 1 \mathrm{H}, 4-\mathrm{H}\right), 4.51(\mathrm{~d}, 1 \mathrm{H}, 3-\mathrm{H}), 4.53,4.62 \\
\left.\text { (AB syst., } J_{1,1}=-19.5 \mathrm{~Hz}, 2 \mathrm{H}, 1-\mathrm{H}\right)\end{array}$ \\
\hline 5e & $\begin{array}{l}\left.4.33 \text { (dddd, } J_{\mathrm{F}, 4}=15.9 \mathrm{~Hz}, J_{4.5}=6.2 \mathrm{~Hz}, J_{4.5}=5.0 \mathrm{~Hz}, J_{3,4}=2.1 \mathrm{~Hz}, 1 \mathrm{H}, 4-\mathrm{H}\right), 4.49(\mathrm{~d}, 1 \mathrm{H}, 3-\mathrm{H}) \\
\left.\left.4.58 \text { (ddd, } J_{5,5}=-9.7 \mathrm{~Hz}, J_{\mathrm{F}, 5}=46.6 \mathrm{~Hz}, 1 \mathrm{H}, 5-\mathrm{H}\right), 4.65 \text { (ddd, } 1 \mathrm{H}, 5-\mathrm{H}\right), 4.56,4.66 \text { (AB syst., } \\
\left.J_{1,1}=-19.5 \mathrm{~Hz}, 2 \mathrm{H}, 1-\mathrm{H}\right)\end{array}$ \\
\hline $\mathbf{5 f}$ & $\begin{array}{l}0.93\left(\mathrm{t}, J_{5,6}=7.4 \mathrm{~Hz}, 3 \mathrm{H}, \mathrm{CH}_{3}\right), 1.58\left(\mathrm{~m}, 2 \mathrm{H}, \mathrm{CH}_{2}\right), 3.89\left(\mathrm{ddd}, J_{4,5}=7.4 \mathrm{~Hz}, J_{4,5}=6.6 \mathrm{~Hz}, 1 \mathrm{H},\right. \\
4-\mathrm{H}), 4.34\left(\mathrm{~d}, J_{3,4}=2.3 \mathrm{~Hz}, 1 \mathrm{H}, 3-\mathrm{H}\right), 4.48,4.58\left(\mathrm{AB} \text { syst., } J_{1,1}=-19.4 \mathrm{~Hz}, 2 \mathrm{H}, 1-\mathrm{H}\right)\end{array}$ \\
\hline $5 \mathrm{~g}$ & $\begin{array}{l}\left.2.87(\mathrm{~d}, 2 \mathrm{H}, 2-\mathrm{H}), 4.43\left(\mathrm{~d}, J_{3.4}=2.3 \mathrm{~Hz}, 1 \mathrm{H}, 4-\mathrm{H}\right), 4.48 \text { (ddd, } 1 \mathrm{H}, 3-\mathrm{H}\right), 4.58,4.68 \text { (AB syst. } \\
\left.J_{6.6}=-19.6 \mathrm{~Hz}, 2 \mathrm{H}, 6-\mathrm{H}\right)\end{array}$ \\
\hline
\end{tabular}

13. Bednarski, M.D.; Simon, E.S.; Bischofberger, N.; Fessner, W.-D.; Kim, M.-J.; Lees, W.; Saito, T.;

Waldmann, H.; Whitesides, G.M. J. Am. Chem. Soc. 1989, 111, 627-635.

14. Straub, A. Dissertation, Univ. Stuttgart 1989.

15. Jones, J.K.N.; Mitchell, D.L. Can. J. Chem. 1959, 37, 1561-1566.

16. Gorin, P.A.J.; Hough, L.; Jones, J.K.N. J. Chem. Soc. 1955, 2699-2705.

17. Kochetov, G.A. Methods Enzymol. 1982, 90, 209-223.

Datta, A.G.; Racker, E. J. Biol. Chem. 1961, 236, 624-628. 